\title{
Exploitation of the comic strips in French as a foreign language class in Albania
}

\section{[L'exploitation de la Bande Dessinee en classe de FLE en Albanie]}

\author{
Anida Kisi
}

DOI: 10.18355/XL.2020.13.01.08

\begin{abstract}
This paper conducts an analytical reflection on the reality and the perspective of the integration of comic strips into the teaching / learning of FFL in Albania. It supports the hypothesis that the use of the comic strip in FFL class constitutes a didactic means which favors the progression of the linguistic competences while being a source of pleasure and motivation. It also tries to show that the use of comic strip in FFL helps memorization, triggers interaction and develops the critical thinking of the learner.

The theoretical framework of our research is that of a hybrid exploration based on an approach that reconciles semiology and didactics in the exploitation of comics in the course of FFL. We are interested in closely observing the uses, the place and the function it occupies in FFL textbooks and teaching practices in the Albanian FFL landscape. Some of the main topics that our research focuses on are: Does comic strips as a teaching / learning medium promote the development of language skills? How and for what purposes are the comic strips used in FFL? What is the impact of comic strips on the learner? What problems do we encounter while using coming strips? What technical and didactic needs do we have? What training needs (initial and/or ongoing) do we have?
\end{abstract}

Key words: comics, teaching / learning, FFL, language skills, textbook, Albania

\section{Résumé}

Cette communication propose une réflexion analytique relative à la réalité et à la perspective de l'intégration de la BD dans l'enseignement/apprentissage du FLE en Albanie. Nous partons de l'hypothèse que l'exploitation de la BD en classe de FLE constitue un moyen didactique qui favorise la progression des compétences langagières tout en étant une source de plaisir et de motivation, un moyen didactique qui aide la mémorisation, déclenche l'interaction et développe l'esprit critique chez l'apprenant.

Le cadre théorique de notre recherche est celui d'une exploration hybride s'appuyant sur une démarche qui concilie sémiologie et didactique dans l'exploitation de la BD en cours de FLE. Le cadre fixé, nous nous intéressons ensuite à observer de près les usages, la place et la fonction qu'elle occupe dans les manuels de FLE et dans les pratiques d'enseignements en contexte albanais. La BD comme support d'enseignement/apprentissage favorise-t-elle la progression des compétences langagières ? Comment et à quelles fins l'utilise-t-on en FLE ? Quelle est son impact chez l'apprenant? Quelles problématiques y rencontre-t-on? De quels besoins techniques et didactiques dispose-t-on? Quels besoins en termes de formation (initiale et/ou continue)? - Telles sont les questions qui nous préoccupent.

Mots-clés: bande dessinée, enseignement/apprentissage, FLE, compétences langagières, manuel, Albanie

\section{Introduction}

L'objectif de notre recherche est d'évaluer l'utilisation de la bande dessinée dans le contexte éducatif albanais. Pour ce faire, nous présenterons tout d'abord un

XLinguae, Volume 13 Issue 1, January 2020, ISSN 1337-8384, eISSN 2453-711X 
panorama général ayant trait à la place qui est accordée à la BD en tant que forme d'expression artistique en Albanie. Après avoir présenté brièvement le cadre institutionnel et les standards qui déterminent le choix des manuels de FLE dans le système pré-universitaire albanais, nous effectuerons une étude qualitative de notre corpus, constitué d'exemples de BD faisant partie du manuel «Le Mag' » $(1,2,3)$, de l'éditeur Hachette. Notre étude n'étant pas exhaustive, nous avons opté pour restreindre notre recherche aux manuels didactiques utilisés au collège, à savoir de la $6^{\text {ème }}$ à la $9^{\text {ème }}$ classe.

\section{Pourquoi «Le Mag'»?}

En premier lieu, à cause de l'emploi fréquent et systématique de la BD dans ce manuel, et en second lieu parce qu'il s'agit de l'un des manuels les plus longuement et massivement utilisés dans les collèges albanais. Notre analyse est articulée en deux parties. Dans un premier temps, nous analyserons les différentes activités et compétences visées au travers des extraits de BD dans le manuel. Ensuite, nous effectuerons une analyse du discours des entretiens réalisés avec des enseignants. Les questions portent sur la manière dont les cours se déroulent, mais également sur les difficultés rencontrées et sur l'opinion des enseignants à l'égard de ce support.

\section{La BD et le lecteur albanais}

La Bande Dessinée (BD) ${ }^{1}$ n'est pas méconnue en tant que forme artistique d'expression pour le public albanais, mais elle est fort loin de jouir de la réputation et du statut qui lui sont reconnus dans le monde francophone. Force est de constater que malgré la présence d'illustrateurs authentiquement doués en Albanie, la narration accompagnée de dessins ou les récits illustrés est rangée dans un coin à l'écart de l'univers artistique albanais, et est associée à un support servant surtout à amuser les enfants et les adolescents. Cette forme d'expression a connu sa plus grande popularité auprès du jeune lectorat albanais pendant la période communiste, notamment dans les années 70-80 grâce à la publication du magazine «Pionieri » (Le Pionnier), lequel s'adressait à un public d'adolescents de 10-14 ans, que l'on appelait à l'époque les « pionniers du parti communiste ${ }^{2}$.

Le contenu de ce magazine, qui se définissait comme politico-social, littéraire et artistique, technique et scientifique, était fortement imprégné de nuances politiques, comme d'ailleurs tous les médias de l'époque. Le magazine contenait également «des récits de trois ou quatre pages présentées sous le format de la bande dessinée, mais bien différents de celles-ci (absence des bulles [...], monotonie des dessins, absence d'interjections) » (Dado, 2015).

D'ailleurs, la première exposition d'illustrations albanaises depuis la constitution de l'Etat albanais (1912) a seulement eu lieu en avril 2018. Les allocutions d'ouverture déploraient un manque d'attention à leur égard, en reconnaissant la place marginale accordée jusqu'alors aux artistes et à leurs productions artistiques. Gazmend Leka avouait ainsi dans son allocution :

\footnotetext{
${ }^{1}$ Le principe commun à toutes les définitions proposées par différents théoriciens de la bande dessinée (McCLOUD 1993, GROENSTEEN 2006, FILIPPINI 2005, BI 2006) est qu'elle est une manière de raconter une histoire à l'aide d'images successives.

${ }^{2}$ En albanais : Pionieri organ i KQ të BRPSH. Revistë e përdyjavshme politike-shoqërore, letrare-artistike, teknike-shkencore për pionierët e ciklit të lartë të shkollës tetëvjeçare.
} 
"Notre école d'arts n'a pas eu d'atelier spécifique pour les illustrateurs. Il ont choisi eux-mêmes de le devenir, à titre de vocation personnelle et d'une année à l'autre, ils ont eux-mêmes bâti l'école albanaise de l'illustration ».'

En mars 2012, dans le cadre de la Francophonie, l'auteur des bandes dessinées Kek arrivait en Albanie pour initier les jeunes et les moins jeunes à la BD. Il affirme à son retour :

"C'est un art qui est vraiment très peu connu là-bas. Il y a quelques $B D$, mais pour les gens, ce ne sont que des trucs pour les enfants. Du coup, je me suis rendu dans des écoles, collèges, lycées, pour donner des cours de dessins et de BD. C'était super intéressant, et ils étaient bien motivés. Si un jour j'apprends qu'un Albanais fait de la BD grâce à moi, je serai super fier. " ${ }^{4}$

Le dessin dans la littérature de jeunesse albanaise sert donc plutôt à illustrer; il n'acquiert jamais le statut d'une composante qui participerait à la narration, comme dans le cas de la BD. De plus, les dessins, pour les Albanais, sont majoritairement associés soit à l'humour (dessins satiriques et caricatures), soit aux contenus puérils. Cela nous amène à avouer que jusqu'à ce début du troisième millénaire, la BD ne représente pas pour les Albanais une forme d'expression artistique ou littéraire à part entière, et les manuels scolaires albanais ne l'exploitent que très rarement à des fins d'enseignement/apprentissage. La BD demeure peu ou pas connue par le public albanais, la source principale du contact avec cette forme d'art restant les livres ou manuels en langue étrangère.

Cependant, maintes initiatives, soutenues notamment par l'Ambassade de France et différents acteurs de la culture en Albanie, visent à revitaliser et à donner un souffle réel au développement du neuvième art (Huerta, 2005). Une série d'activités organisées en collaboration avec le Ministère de la Culture ont visé à promouvoir auprès du grand public les formes d'expression artistique autour des arts graphiques. Du 28 mai au 2 juin 2018 fut organisée la Semaine de découverte autour de la Bande Dessinée. A l'invitation de l'Ambassade de France, et en partenariat avec le Ministère de la Culture albanais ainsi que le réseau des Alliances françaises en Albanie, deux dessinateurs français de bandes dessinées, Cédric Fortier et Marine Blandin, ont parcouru l'Albanie pour faire découvrir leur travail et l'univers de la BD française. Avec douze ateliers dans six villes, ils ont eu l'occasion de rencontrer plus de deux cent vingt dessinateurs amateurs et professionnels, et de partager ainsi avec eux leur passion.

Le 15 novembre 2018 à Tirana, l'Ambassade de France en Albanie était partenaire du Ministère de la Culture albanais pour le Prix National de la Bande Dessinée. Le lauréat était en résidence du $1^{\mathrm{er}}$ au 15 décembre à la Cité internationale de la bande dessinée et de l'image. Cette activité a fait suite au spectacle de fin d'année du réseau des Alliances françaises organisé avec l'Ambassade de France en Albanie. Du 10 au 14 décembre 2018, Le dessinateur albanais David Kryemadhi, le dessinateur français Cédric Fortier, et les musiciens du Hot Club Tirana ont parcouru

\footnotetext{
${ }^{3}$ Gazmend Leka : «Shkolla jonë e arteve, nuk pati një atelie të veçantë për ilustratorët. Ata zgjodhën vetë të ishin të tillë, si një thirrje vetjake dhe vit pas viti, e ndërtuan po vetë ata shkollën e ilustrimit shqiptar. Disponible en ligne : https://gazetamapo.al/ta-rivitalizojneilustrimin-shqiptar / 04/04/18.

${ }^{4}$ Interview du dessinateur KEK. Source : https://shunrize.com/blog/les-annees-college-kek/ consultée en ligne le 10/10/2019.
}

XLinguae, Volume 13 Issue 1, January 2020, ISSN 1337-8384, eISSN 2453-711X 
l'Albani. Les spectateurs albanais ont ainsi pu assister à une forme inédite mêlant le jazz à la bande dessinée : le concert dessiné.

Pour conclure cette première partie de notre réflexion, nous tenons à mettre en exergue ce fait que les apprenants de la langue française figurent parmi ceux qui ont une idée suffisamment claire de ce que c'est la BD : ils ont rencontré cette forme d'expression dans leur parcours d'apprentissage de par la place qui est accordée par les manuels de FLE aux documents authentiques en général, et particulièrement à la Bande Dessinée. La majorité des manuels de FLE de ces dernières années sont remplis d'illustrations et de photographies utilisées pour aborder différents contenus de manière attractive et ludique, afin de développer les compétences communicatives et interculturelles.

\section{Cadre institutionnel}

Depuis 2006, l'Albanie est membre de l'Organisation Mondiale de la Francophonie. A ce titre, et dans le respect du plurilinguisme, les décideurs nationaux s'engagent à maintenir au niveau national un pourcentage de $30 \%$ d'élèves qui vont continuer à apprendre le français à l'école (Vishkurti, 2010). Les réformes des dernières années et le nouveau curriculum, inspirés par les programmes occidentaux, mettent en avant la valorisation des processus d'apprentissage et la méthodologie transdisciplinaire selon la logique de compétence (Arapi, Lassere, 2016 ). Cela a évidemment favorisé la diversification des outils pédagogiques en didactique des langues, et notamment du FLE. De plus, dans le Cadre Européen Commun de Référence pour les Langues (CECRL), qui constitue une base de référence pour l'enseignement de langues étrangères en Albanie, le terme "Bande Dessinée » apparaît dans la section des différents types de textes que les apprenants peuvent recevoir, produire ou échanger (Conseil de l'Europe, 2001). La bande dessinée est donc un outil communicatif respectant les nouvelles méthodes d'apprentissage des langues.

La procédure de sélection des manuels FLE est sanctionnée par la loi et les ordonnances du Ministère. Chaque année, les maisons d'édition nationales présentent leurs propositions aux commissions officielles d'évaluation. En ce qui concerne la langue française, la majorité des propositions sont des manuels d'éditeurs français prestigieux tels que Hachette, Clé International, etc. Ils sont soumis à une procédure d'évaluation à l'aveugle basée sur une grille d'évaluation (de standards et d'indicateurs).

Les manuels qui obtiennent les meilleures évaluations sont proposés aux écoles, dans lesquelles le choix décisif se fait par l'équipe pédagogique en présence des parents. La pluralité des manuels et leur procédure de sélectiontémoignent de la démocratisation et de l'ouverture des méthodes contemporaines d'enseignement.

Pour la langue française, les titres proposés aux écoles par le Ministère, pour le collège et le lycée :

1. Le Kiosque 2, Hachette.

2. Le Mag' 1, Hachette.

3. Decibel 2, Les Editions Didier.

4. Mot de passe 2, Educational Centre Trait d'union.

5. Pourquoi pas! 4, Uegen Editions.

6. Adosphère, Hachette.

7. Appli ado, Educational Centre Trait d'union.

8. Mot de passe, Educational Centre Trait d'union. 
Nous constatons que la présence des $\mathrm{BD}$ dans un manuel contribue à satisfaire plusieurs standards exigés par le Ministère de l'Education (MAS, 2015 : 39-40) tels que :

- Le développement des compétences de communication et d'expression en langue étrangère (Std. 16.1.3.2)

- L'équilibre entre les compétences de compréhension orale, écrite, et l'expression orale et écrite (Std. 16.1.3.3)

- $\quad$ L'information sur la culture du pays concerné (16.1.3.)

- $\quad$ Le manuel, à travers sa langue de photographies et d'illustrations, favorise l'éducation interculturelle (Std. 16.1.3.10)

- $\quad$ Le manuel contribue à la connaissance de la culture du pays en ce qui concerne les aspects interculturels (Std. 16.1.3.12)

- $\quad$ Le manuel présente des situations (exemples, exercices, questions et réponses, tâches) qui facilitent le travail indépendant et actif des étudiants dans le processus d'apprentissage (Std. 16.1.3.14)

- $\quad$ Le manuel propose des situations (exemples, exercices, questions) qui développent l'imagination et la créativité des élèves (Std. 16.1.3.16)

- $\quad$ Le manuel fournit des exemples sur la façon d'apprendre. L'élève doit faire l'expérience de ce qu'il apprend et réfléchir par le jeu ou par différents rôles, dans la classe et dans la vie quotidienne (Std. 16.1.3.17).

\section{Présentation du manuel « Le Mag'»}

«Le Mag'» se présente sous la forme d'un magazine créé par quatre adolescents participant à l'atelier de journalisme de la Maison des Jeunes de leur quartier. Le style " magazine » du manuel a par ailleurs pour atouts d'être visuellement attrayant et de proposer des documents très variés. Pour chaque unité, des documents écrits semi-authentiques (reportages, courriers des lecteurs, interviews, tests...) offrent la possibilité de travailler, toujours en contexte, les autres contenus de l'unité. Ces documents mènent à des situations de communication, la plupart du temps écrites. Les exercices de systématisation grammaticale sont réunis dans la page Atelier Langue afin de préserver l'aspect communicatif et contextuel des premières doubles pages. Pour clore chaque unité, la page bilan de Fais le point reprend les contenus de l'unité et permet de travailler les quatre compétences pour se préparer à l'examen du Nouveau DELF niveau A1 (version junior). Elle donne également l'occasion aux élèves d'évaluer leurs progrès et de faire le point sur leurs acquis. Enfin, l'esprit magazine est présent pour aborder des thèmes culturels propres à la France, dans les pages Civilisation que l'on retrouve toutes les deux unités. Des activités interculturelles y sont également proposées afin d'encourager les élèves à réfléchir aux similarités ou différences observées entre la culture française et la leur. 


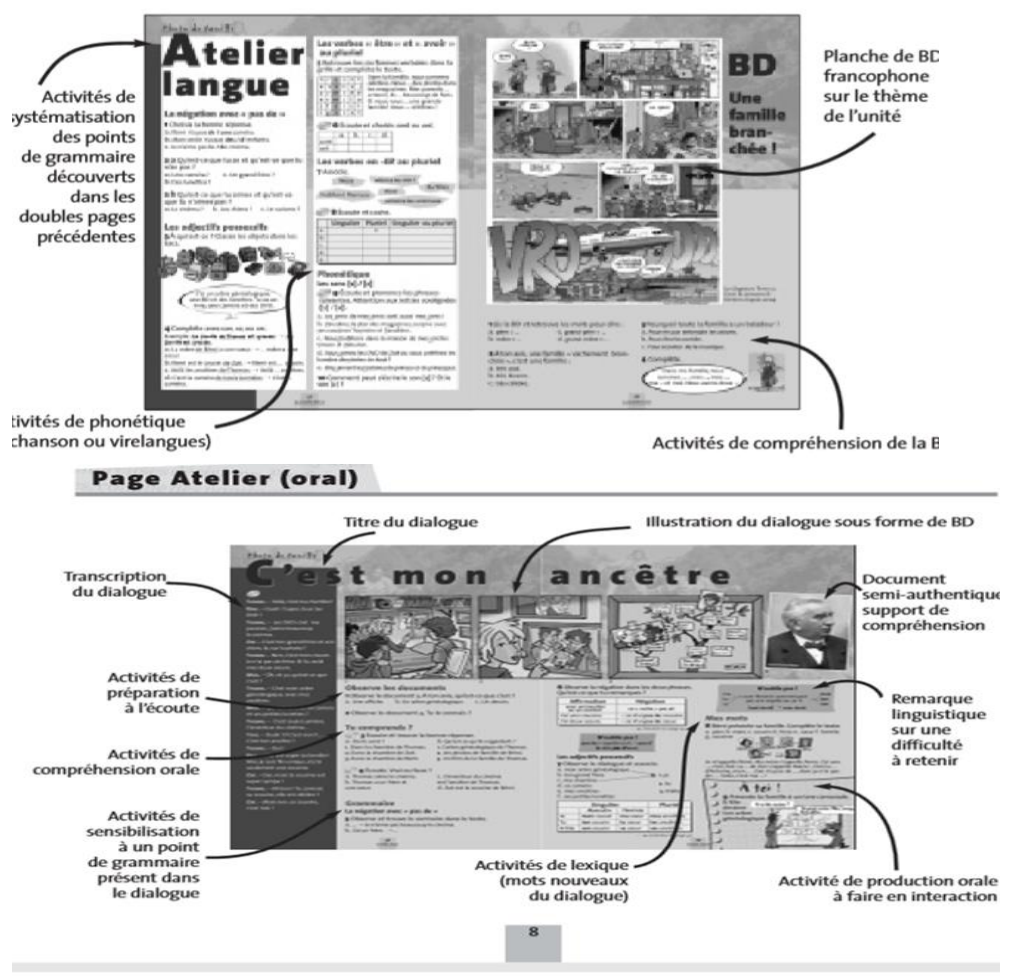

Structure d'une unité

\section{Guide pédagogique « Le Mag' 1 » p. 8-9}

Une planche de bande dessinée authentique, la page BD, adaptée à la fois au thème et aux contenus de l'unité, présente des héros de la bande dessinée francophone et enrichit le lexique d'un vocabulaire plus familier. Elle contribue à l'aspect magazine de la méthode. Le tableau suivant permet de connaître en détail certaines données sur les séquences de BD du manuel, ainsi que les activités et les modalités de travail qui les concernent.

Tableau de présentation des BD, des activités et des modalités de travail pour les manuels « Le Mag' » 1,2 et 3

\begin{tabular}{|c|c|c|c|}
\hline \multicolumn{4}{|c|}{ Le Mag 1} \\
\hline & BD & Auteur & Activités didactiques \\
\hline $\begin{array}{l}\text { Unité } \\
1, \mathrm{BD} \\
\text { p.17 }\end{array}$ & $\begin{array}{ll}\text { Tony } & \text { et } \\
\text { Alberto } & \end{array}$ & Dab's, & $\begin{array}{l}\text { Activités de lecture, de compréhension et } \\
\text { d'expression orale }\end{array}$ \\
\hline $\begin{array}{l}\text { Unité } \\
2, \quad \text { p. }\end{array}$ & Titeuf & Zep & $\begin{array}{l}\text { Découverte du langage familier, activités de } \\
\text { lecture et de compréhension globale }\end{array}$ \\
\hline
\end{tabular}




\begin{tabular}{|c|c|c|c|}
\hline 25 & & & \\
\hline $\begin{array}{l}\text { Unité } \\
3, \quad \text { p. } \\
35\end{array}$ & Rémi 2 & $\begin{array}{l}\text { O. Balez } \\
\text { et L. } \\
\text { Richard }\end{array}$ & $\begin{array}{l}\text { Activités de compréhension et d'expression, } \\
\text { Jeu de rôles }\end{array}$ \\
\hline $\begin{array}{l}\text { Unité } \\
4, \quad \text { p. } \\
43\end{array}$ & Les Zappeurs & $\begin{array}{l}\text { Ernstet } \\
\text { Janssens }\end{array}$ & $\begin{array}{l}\text { Activités de lecture, de compréhension, } \\
\text { lexique }\end{array}$ \\
\hline $\begin{array}{l}\text { Unité } \\
5, \quad \text { p. } \\
53\end{array}$ & Les Zappeurs & $\begin{array}{l}\text { Ernstet } \\
\text { Janssens }\end{array}$ & $\begin{array}{l}\text { Activités de lecture, de compréhension, } \\
\text { reformulation }\end{array}$ \\
\hline $\begin{array}{l}\text { Unité } \\
6, \quad \text { p. } \\
61\end{array}$ & Boule et Bill & $\begin{array}{l}\text { Dargaud- } \\
\text { Lombard }\end{array}$ & Activités de compréhension \\
\hline $\begin{array}{l}\text { Unité } \\
7, \quad \text { p. } \\
71\end{array}$ & $\begin{array}{l}\text { Magazine } \\
\text { Astrapi }\end{array}$ & $\begin{array}{l}\text { Marc } \\
\text { Cantin }\end{array}$ & $\begin{array}{l}\text { Compréhension globale, travail sur le } \\
\text { lexique }\end{array}$ \\
\hline $\begin{array}{l}\text { Unité } \\
8, \quad \text { p. } \\
79\end{array}$ & Titeuf & Zep & $\begin{array}{l}\text { Lecture } \\
\text { Expression orale }\end{array}$ \\
\hline
\end{tabular}

\section{Le Mag 2}

\begin{tabular}{|c|c|c|c|c|}
\hline $\begin{array}{l}\text { Unité } \\
2, \quad \text { p. } \\
25\end{array}$ & $\begin{array}{l}\text { Franky } \\
\text { Snow }\end{array}$ & Buche & $\begin{array}{l}\text { Lecture individuelle } \\
\text { Compréhension globale } \\
\text { Lexique familier } \\
\text { Correction collective }\end{array}$ & Par deux \\
\hline $\begin{array}{l}\text { Unité } \\
4, \quad \text { p. } \\
43\end{array}$ & Smala 2 & $\begin{array}{l}\text { M.Paulo et } \\
\text { T. } \\
\text { Robberecht. }\end{array}$ & $\begin{array}{l}\text { Observation } \\
\text { Lecture } \\
\text { Expression } \\
\text { Travail sur le } \\
\text { vocabulaire } \\
\text { Résumé } \\
\text { Prononciation, } \\
\text { intonation }\end{array}$ & $\begin{array}{l}\text { Par deux, } \\
\text { individuellement }\end{array}$ \\
\hline $\begin{array}{l}\text { Unité } \\
6, \quad \text { p. } \\
61\end{array}$ & $\begin{array}{l}\text { Léo et } \\
\text { Luestune }\end{array}$ & Jak et Geg & $\begin{array}{l}\text { Observation } \\
\text { Lecture } \\
\text { Expression }\end{array}$ & $\begin{array}{l}\text { Individuellement } \\
\text { Par deux } \\
\text { Mise en commun }\end{array}$ \\
\hline $\begin{array}{l}\text { Unité } \\
7, \quad \text { p. } \\
71\end{array}$ & Smaxestune & $\begin{array}{l}\text { Christophe } \\
\text { Bertschy }\end{array}$ & $\begin{array}{l}\text { Phase d'observation } \\
\text { Lecture } \\
\text { expression }\end{array}$ & $\begin{array}{l}\text { Individuellement } \\
\text { Mise en commun }\end{array}$ \\
\hline
\end{tabular}

\section{Le Mag 3}

\begin{tabular}{|l|l|l|l|l|}
\hline $\begin{array}{l}\text { Etape } \\
4\end{array}$ & Toto & $\begin{array}{l}\text { Thierry } \\
\text { Coppée }\end{array}$ & $\begin{array}{l}\text { Phase d'observation } \\
\text { Lecture/Compréhension } \\
\text { Expression }\end{array}$ & $\begin{array}{l}\text { Individuellement } \\
\text { Mise en commun }\end{array}$ \\
\hline
\end{tabular}

Nous constatons que la BD est généralement analysée en trois étapes : avant la lecture, pendant la lecture, et après la lecture. Dans l'étape d'avant lecture, il s'agit XLinguae, Volume 13 Issue 1, January 2020, ISSN 1337-8384, eISSN 2453-711X 
plutôt de faire faire un remue-méninge. L'objectif est d'activer les connaissances antérieures ou extralinguistiques des apprenants. L'étape pendant la lecture contient les activités de compréhension, de réflexion et de discussion. Il s'agit de l'analyse complète du document. L'étape d'après lecture peut être vue aussi comme une étape d'évaluation. C'est l'étape qui permet de voir ce que l'apprenant peut faire après avoir travaillé avec un certain document.

L'exploration du manuel «Le Mag “» démontre que la bande dessinée insérée dans le manuel est utilisée en premier lieu comme déclencheur d'interactions. Elle ouvre chaque unité et incite la participation des élèves par le biais des activités d'observation, de repérage et de compréhension globale. Par la suite, à la fin de chaque unité dans le manuel «Le Mag'1, 2, 3 » les planches de BD sont suivies d'activités visant :

La compréhension globale: Vrai ou faux ?/ Questions fermées/ QCM sur le contenu de la BD, etc.

- Le lexique: Associer/Trouver les expressions pour dire.../ Trouver les synonymes de..., Repérer les mots et expressions du registre familier, etc.

- $\quad$ L'expression orale : Imagine la suite de la journée de.../Questions ouvertes : Pourquoi ? A ton avis... ? Exprimer une opinion sur ..., Jeux de rôle, etc.

- $\quad$ La grammaire : Retrouver le participe passé, etc.
La prononciation et l'intonation.

Même si les possibilités d'utilisation en classe sont multiples, il peut s'agir tout d'abord de comprendre une bande dessinée globalement à partir de l'observation de l'image, du dessin, et de réfléchir sur les procédés graphiques. Après, on peut entraîner à la compréhension écrite et à l'expression orale en demandant de raconter l'histoire selon différents points de vue, trouver un titre, imaginer ce qui s'est passé avant, ce qui se passera après, imaginer le contenu des bulles, trouver le mot de la fin, manipuler certaines structures grammaticales et des actes de parole en situation authentique. Ensuite, on peut passer à l'expression orale pure, en développant la créativité des apprenants (Morlat et Tomimoto, 2004 : 52-54).

\section{Repères théoriques sur le potentiel éducatif de la BD}

Plusieurs recherches ${ }^{5}$ témoignent du potentiel éducatif de la bande dessinée et nombre de spécialistes ${ }^{6}$ se sont déjà penchés sur les possibilités éducatives du langage narratif verbo-iconique. Pour être en mesure de comprendre le message en lisant une bande dessinée, le lecteur doit conjuguer les images et le texte. La bande dessinée est une lecture particulière parce que le lecteur doit en synthétiser les différents éléments pour comprendre le message. L'image précise et renforce le texte ; l'information est mise en récit. Dans la BD, il y a une complémentarité entre le visuel et le verbal : c'est un langage cohérent dans le cadre duquel ces deux éléments se lisent de façon indissociable.

\section{Le rapport texte-image}


Il est important de s'interroger sur cette cohabitation entre le texte et l'image, ou de mieux définir la relation entre code iconique et code linguistique qu'ils entraînent au sein d'un même message. Étant dans une relation complémentaire lorsqu'ils sont utilisés dans un même message, ils constituent rarement des éléments distants l'un de l'autre. On observe une relation étroite entre l'image et le texte. Pour Roland Barthes (1964 : 40-51), cette liaison semble être apparue dès que le livre est né. Barthes considère que la communication propose un lien permanent entre texte et image, et que le message linguistique qui accompagne l'image joue deux rôles possibles : un rôle d'ancrage et un rôle de relais.

\section{Le rôle d'ancrage}

Essentiellement sous forme de légende, titre, accroche ou signature, la fonction de l'accompagnement linguistique de l'image tend à réduire sa polysémie, " elle arrête la chaîne flottante des signifiés " (Joly, 2015 : 90). Cette fonction guide, fixe, arrête, parmi toutes les significations possibles, un sens, en empêchant l'interprétation subjective. Le titre, la légende, l'accroche, la signature, dictent le sens unique de lecture de l'image. La langue est ici en position de force par rapport à l'image.

Selon Martine Joly la fonction d'ancrage consiste à arrêter cette chaîne flottante du sens qu'engendrerait la nature polysémique de l'image, en désignant quoi privilégier parmi les différentes interprétations que peut solliciter l'image seule. Ainsi, elle désigne par cette fonction l'idée que le texte peut réduire la polysémie de l'image, que l'image peut avoir plusieurs significations mais qu'elle peut aussi ancrer à son tour le texte. En l'illustrant et en accompagnant celui-ci, elle dirige par là sa lecture.

\section{Le rôle de relais}

Le message linguistique permet de rajouter au message iconique des significations que ce dernier ne peut pas, ou qui lui sont difficiles à transmettre. Malgré la qualité expressive ou esthétique d'une image, elle ne peut tout dire sans avoir recours au verbal. La fonction de relais oriente la polysémie de l'image et permet d'en préciser le sens.

La fonction de relais, telle que l'a définie Roland Barthes (1964 : 40-51), est une forme de complémentarité entre l'image et les mots. L'image fixe est incluse dans le texte; elle ne peut pas raconter une histoire ou parler d'elle-même sans l'intervention de la langue. Un bon exemple de fonction de relais dans les images fixes sont les bandes dessinées, les images en couverture d'un livre, les dessins qui accompagnent certains textes au niveau desquels il y a un rapport de complémentarité entre texte et image.

Madeleine Gauthier explique le rôle que l'image et la BD peuvent jouer dans les différents apprentissages : "Bien avant de savoir déchiffrer les mots, les enfants apprennent à déchiffrer l'image : ils reconnaissent les mécanismes et les leviers du déroulement d'une histoire. La bande dessinée fait partie intégrante de leur apprentissage à communiquer ; elle pourrait aussi devenir source de savoir » (Gauthier, 2002, $\mathrm{n}^{\circ} 1$ et 2).

\section{Aspect ludique}

Il peut, dans certains cas, mener à un autre niveau de compréhension de l'information. La bande dessinée est aussi un bon moyen de présenter l'information de manière intéressante et ludique - le ludique pouvant se révéler bénéfique en situation d'apprentissage (MAKHLOUFI, 2011). Elle peut expliquer, sensibiliser et mettre en

XLinguae, Volume 13 Issue 1, January 2020, ISSN 1337-8384, eISSN 2453-711X 
situation l'information, la rendant éducative ou instructive. Lorsqu'elle est intégrée comme outil d'apprentissage dans le milieu scolaire, elle peut également devenir pédagogique (Tremblay, $2013: 1$ ). La combinaison texte-image-séquence, si elle est utilisée et développée adéquatement, peut aider l'apprenant à comprendre, à réfléchir et, éventuellement, à mieux retenir l'information. La bande dessinée éducative pourrait être considérée dans le cadre de la diversification des méthodes d'apprentissage.

De plus, Aydoğu (2015) confirme que: «Par l'association texte-image, la bande dessinée permet de donner suffisamment d'indices aux apprenants pour la compréhension globale d'un texte et éveille la lecture active. Ainsi elle permet une entrée plus facile dans la lecture autonome et elle devient lecture plaisir ».

Par l'association texte-image, la bande dessinée permet de donner suffisamment d'indices aux apprenants pour la compréhension globale d'un texte, et éveille la lecture active. Ainsi, elle permet une entrée plus facile dans la lecture autonome et elle devient lecture plaisir.

\section{Motivation et attention}

L'attention et la motivation sont des éléments importants de la théorie d'apprentissage (le modèle de l'autorégulation, Zimmerman, 2002). Selon cette théorie, le rôle de l'enseignant serait d'améliorer la motivation des élèves avec des stratégies qui augmentent l'attention, qui améliorent la pertinence, qui encouragent la confiance et garantissent la satisfaction (DRISCOLL 2005 : 421). La bande dessinée pourrait répondre à ces critères. La bande dessinée se présente comme un outil d'apprentissage différent, pouvant augmenter l'attention des élèves vis-à-vis la matière à apprendre. De plus, elle permet d'augmenter leur motivation à l'apprentissage et à la lecture en général. La présentation d'information par la bande dessinée, en plus d'avoir un impact potentiel sur l'attention et la motivation, pourrait également influencer la manière dont l'apprenant perçoit cette information (TUNCEL et AYVA, 2010). Djamel ( 2007 ) souligne que « la BD par son aspect distrayant et ludique peut aider une classe de langue à mieux communiquer dans une langue étrangère. Nous ne prétendons pas innover en la matière; nous voulons simplement dire que sa présence en classe avec les diverses activités auxquelles elle se prête peut amener une certaine détente, sans oublier l'enrichissement culturel dont vont profiter les apprenants ».

\section{Valeurs interculturelles}

De plus, l'apprentissage d'une langue étrangère coïncidant avec la connaissance d'une ou plusieurs autres cultures, il donne accès à d'autres usages, à d'autres modes de pensée, à d'autres valeurs. En plus de développer des compétences langagières, la bande dessinée, produit culturel, permet de lever certaines idées ou certains jugements négatifs que peut porter l'apprenant sur l'autre. Mireille Carton met l'accent sur cette double fonction de la $\mathrm{BD}$ en classe de français : « Le F.L.E, français langue étrangère, utilise souvent la BD pour travailler sur la langue et aussi sur les représentations et les stéréotypes. » (Tremblay, 2013). En tant que document authentique, la bande dessinée pourrait susciter des débats qui permettent d'aboutir à des opinions positives ou négatives, ou même les deux ensemble (Tagliante, 1994).

\section{Points de vue des enseignants du FLE et conclusions}

Les enseignantes interviewées sont au nombre de quatre et enseignent au sein de collèges de Tirana, capitale de l'Albanie. Les interviewées sont des femmes et ont plus de 35 ans. Les questions portent sur la manière dont les cours se déroulent, mais également sur les problématiques auxquelles elles se heurtent lors de l'utilisation des bandes dessinées en classe de FLE. 
Les entretiens révèlent que la BD est rarement utilisée en classe de FLE dans les collèges albanais. Même dans les cas où l'on y a recours, son exploitation est assez superficielle et se suffit à des simples activités d'observation et de lecture. Les arguments en sa défaveur sont: le manque de temps, le nombre d'élèves (qui varie entre 35 et 40 élèves par classe, et qui rend le travail et le contrôle des élèves très difficiles), et la priorité donnée aux activités centrées sur la grammaire et le lexique. Pour les enseignantes l'apport de la BD dans l'acquisition des règles syntaxiques et grammaticales ou pour la conjugaison n'est pas si évident étant donné que, souvent, on y utilise des tournures et du lexique familier.

Toutes les enseignantes questionnées sont convaincues de l'efficacité de la BD comme outil de communication présentant une situation d'interaction authentique pouvant motiver les élèves, d'autant plus que la participation en classe demeure insuffisante. Quelques cinq ou six élèves par classe de 35 à 40 essaient de suivre le cours en participant ; le reste ne fait que répéter les différentes réponses données par leurs camarades, et il est très difficile de les faire parler.

Les enseignants nous ont confié que la BD n'a jamais fait l'objet de séminaires pédagogiques ou de formation. Les interviewées déclarent également ne pas avoir pris pour support didactique une bande dessiné en dehors de celles contenues dans le manuel. Elles expriment le besoin et l'intérêt d'être formées adéquatement afin de pouvoir intégrer efficacement la bande dessinée dans leur classe du FLE.

Les enseignants pensent que la bande dessinée est susceptible d'être utilisée pour réaliser en classe des activités de compréhension à l'écrit, à la lecture, de vocabulaire et d'expression orale/écrite. De plus, grâce à la $\mathrm{BD}$, l'élève s'ouvre sur d'autres cultures et mentalités. Les enseignants sont conscients que la BD est un support assez ludique, qui suscite l'intérêt des élèves.

Toutes les enseignantes sont ouvertes et prêtes à multiplier ainsi qu'à faire évoluer les techniques et méthodologies de la transmission du savoir au sein de nos environnements d'enseignement. Elles considèrent que la progression de l'enseignant vers les nouvelles technologies, supports et méthodes d'enseignement, est aussi importante que la progression de l'apprenant. Dans ce contexte, la bande dessinée constitue un bon support pour accéder aux savoirs en français langue étrangère. Elle permet la progression des compétences, la motivation et l'encouragement des apprenants, tout en stimulant leur réussite à l'école.

En guise de conclusion de notre étude, nous pouvons affirmer que l'intégration de la $\mathrm{BD}$ à des fins d'apprentissage en classe de FLE est presque inexistante dans le système éducatif pré-universitaire albanais. Cela pourrait être expliqué tout d'abord par la méconnaissance de la BD en tant que forme d'expression artistique au niveau national. Ajoutons à cet argument le fait que l'apprentissage d'une langue étrangère dans nos collèges se réduit le plus souvent à l'étude du lexique et de la grammaire, et que tout travail avec un document authentique est souvent considéré comme un véritable exploit. N'oublions pas, pourtant, que cette attitude méfiante envers la BD est également présente dans le monde francophone, et Nicolas Rouvière confirme que " la bande dessinée a longtemps eu mauvaise presse auprès des milieux éducatifs " (2012 : 7). Les entretiens avec des enseignants nous permettent cependant de d'espérer un accroissement d'attention envers cet art media-culturel (MAIGRET et STEFANELLI, 2012). N'oublions pas pour autant que « les documents authentiques sont des documents bruts élaborés par des francophones pour des francophones à des fins de communication (...) et non en vue de l'apprentissage d'une langue. » JeanPierre Cuq et Isabelle Gruca (2005 : 431). On pourrait en déduire que leur utilisation à des fins didactiques, surtout dans un milieu allophone comme celui de l'albanais, nécessite la formation continue du corps enseignant: celle-ci s'avère en effet essentielle pour appréhender et intégrer au mieux, dans les processus d'enseignement

XLinguae, Volume 13 Issue 1, January 2020, ISSN 1337-8384, eISSN 2453-711X 
et d'apprentissage, les pratiques ou les codes culturels et sociaux, ainsi que les différentes productions artistiques du peuple dont on étudie la langue.

\section{Bibliographic references}

ARAPI, E. - LASSERRE F. 2016. Le cycle de réformes éducatives en Albanie : une transition vers les normes occidentales? Revue internationale d'éducation de Sèvres, Available online: http://journals.openedition.org/ries/5492 ; DOI : 10.4000/ries.5492

BARTHES, R. 1964. Rhétorique de l'image. In: Communications, 4, Recherches sémiologiques. pp. 40-51. DOI : https://doi.org/10.3406/comm.1964.1027 Available online: https://www.persee.fr/doc/comm_0588-8018_1964_num_4_1_1027

AYDOGU, C. 2015. La bande dessinée: un exemple d'exploitation en classe de FLE. Balıkesir University. The Journal of Social Sciences Institute. vol. 18, n. 33.

BOUTIN, J.-F. 2005. Enseigner la syntaxe française au moyen de la bande dessinée: une recherche-développement en formation initiale des maîtres, Nouveaux cahiers de la recherche en éducation, vol. 8, n. 1, pp.111-130.

CONSEIL DE L'EUROPE. 2001. CECRL, chapitre 4.6 le texte. Strasbourg : Didier. $75 \mathrm{p}$.

CUQ, J-P. - GRUCA, I. 2002. Cours de didactique du français langue étrangère et seconde, Grenoble : PUG-FLE.

DADO, F. 2015. Quelques réflexions sur la traduction des interjections dans la bande dessinée, le cas de Tintin, "Le spectre d'Ottocar, Actes du colloque, Pont entre deux rives: la France, l'image de l'occident sous l'optique des intellectuels albanais, Mediaprint.

DJAMEL, B. 2007. La bande dessinée comme support didactique dans l'enseignement du Fle. Synergies Algérie, 1, pp. 235-240.

DRISCOLL, M. P. 2005. Psychologie of learning for instruction, Boston, Pearson Education.

JOLY, M. 2005. Introduction à l'analyse de l'image. Paris. Nathan.

FARID, G. 1980. Exploitation pédagogique de la bande dessinée. Didactique du Français au primaire. Montréal. Les éditions Ville-Marie.

GAUTHIER, M. 1996. La littérature de jeunesse et son pouvoir pédagogique, Education et Francophonie, vol. XXIV n. 1et 2.

GAUTHIER, M. L'apport pédagogique de la bande dessinée, File://A/EFvol_241-2l'apport de la bande dessinée_Art-6htm

GRYSPEERDT, A. 1983. Usages scolaires des médias, Louvain-la-Neuve, Cabay.

GUAY, L. - CHARETTE, D. 2009. La bande dessinée: un outil didactique pour enseigner l'histoire, Revues Traces, vol. 47 (2)

HUERTA, N. 2005. Comment utiliser la bande dessinée comme outil pédagogique à l'école ? I.U.F.M : Académie de Montpellier, site de Carcassonne.

MAIGRET, E. - STEFANELLI, M. (dir.). 2012. La bande dessinée : une médiaculture. Paris : Armand Colin

MINISTERE DE L'EDUCATION ET DU SPORT. 2015. Standarde për tekstet shkollore, kapitulli 16.1.3. Standardi për tekstet shkollore të gjuhëve të huaja, Tiranë, pp. 39-40. Available online: https://arsimi.gov.al/wpcontent/uploads/2015/03/Standardet_tekstet_shkollore1.pdf

JOHNSON, G. C. 2004. Reconceptualising the visual in narrative inquiry into teaching », Teaching and Teacher Education, vol. 20, n. 5, pp.423-434.

LACOSTE, J. H. 1979. Les maîtres du château de Bonaguil et leurs problèmes, Histoire et bande dessinée : acte du 2e Colloque International Éducation et Bande Dessinée, La Roque-d'Anthéron, Objectif Promo-Durance, pp. 44-49.

LAVEAULT, D. - JOLY, R. 1987. La bande dessinée et l'apprentissage de la mathématique au secondaire dans un enseignement par fiches, Revue des sciences de l'éducation, Vol. 13, n. 1, pp. 31-50. 
MORLAT, J.-M. - TOMIMOTO, J. 2004, La bande dessinée en classe de langue, Rencontres Pédagogiques du Kansaï, pp. 52-56, Available online: http://www.rpkansai.com/bulletins/pdf/018/052_056_morlat.pdf

MALLIA, G. 2007. Learning from the sequence: The use of comics in instruction ", ImageTexT: Interdisciplinary Comics Studies, vol. 3, n. 3, Available online: http://imagetext.english.ufl.edu/archives/v3_3/mallia/

MAIGRET, E. - STEFANELLI, M. 2012. (dir.), La Bande dessinée : une médiaculture, Paris, Armand Colin, coll. «Médiacultures ».

MAKHLOUFI, N. 2011. Le ludique dans l'enseignement/apprentissage du FLE chez les 1ère A.S, Synergies Algérie, n. 12, pp. 89-100.

PROST, J. - PELLETIER, A. 1979. « Histoire et bande dessinée », Histoire et bande dessinée : acte du 2e Colloque International Éducation et Bande Dessinée, La Roqued'Anthéron, Objectif Promo-Durance, pp. 26-34.

ROBIN, C. 1977. Perception du langage de la B.D. : son éventuelle utilisation par les enfants, Lecture et bande dessinée : actes du 1er Colloque International Éducation et bande dessinée, Aix-en-Provence, Edicied, pp. 83-89.

ROUVIERE, N. 2012. Bande dessinée et enseignement des humanités, Grenoble : ELLUG

ROUX, A. 1970. La bande dessinée peut être éducative, Paris, Éditions de l'École.

TAGLIANTE, C. 1994. La classe de langue. Paris : CLE International.

TREMBLAY M., 2013. LA BANDE DESSINÉE PÉDAGOGIQUE. Université Laval, Québec, Canada, Available online: https://www.researchgate.net/publication/290448196_Maitrise_sur_mesure_en_desig n_La_bande_dessinee_pedagogique

TUNCEL, G. - AYVA, O. 2010. The utilization of comics in the teaching of the human rights concept, Procedia, Social and Behavioral Sciences 2, pp. 1447-1451

VISHKURTI, S. 2010. Place du français dans le système éducatif albanais, Colloque international: Le français de demain : enjeux éducatifs et professionnels, Sofia, pp.204-2012 Available online : https://crefeco.org/fr_version/pages/8@ Vishkurti.pdf ZIMMERMAN B. 2002. Efficacité perçue et autorégulation des apprentissages durant les études : une vision cyclique. In Carré P., Moisan A. (dir.).La formation autodirigée. Paris : L'Harmattan, pp. 69-88

\section{Available online:}

Interview du dessinateur KEK. Source : https://shunrize.com/blog/les-annees-collegekek/ consultée en ligne le 10/10/2019.

Activités culturelles de l'ambassade de France en Albanie. Source : https://al.ambafrance.org/Semaine-de-decouverte-autour-de-la-Bande-Dessinee, consultée en ligne le 10/10/2019.

Words: 5886

Characters: 38683 (21,49 standard pages)

Dr. Anida Kisi

Department of French Language

Faculty of Foreign Languages,

University of Tirana

Tirana,

Albania

anidakisi@gmail.com 